\title{
Zirconium Zr 89-DFO-cRGDY PEG-Cy5-C' Dots
}

\author{
National Cancer Institute
}

\section{Source}

National Cancer Institute. Zirconium Zr 89-DFO-CRGDY PEG-Cy5-C'Dots. NCI Thesaurus. Code C156739.

A radioconjug ate composed of a tumor-selective fluorescent imaging agent containing silica-based nanoparticles labeled with a near-infrared (NIR) fluorophore, cyanine 5 (Cy5), and surrounded by polyethylene glycol (PEG) chains attached to cyclic arginine-glycineaspartic acid-tyrosine) (cyclo-(Arg-Gly-Asp-Tyr; cRGDY) peptides (C' dots; Cornell prime dots; CRGDY-PEG-C' dots), and linked, via the chelator desferrioxamine (DFO), to the radioisotope zirconium Zr 89 (Zr89), with potential diagnostic properties upon positron emission tomography (PET) imaging. Upon intradermal administration of the zirconium Zr 89 CRGDY PEG-Cy5-C' dots, the CRGDY moiety selectively binds to alphaVbeta3 integ rin expressed on tumor cells. Upon binding, the radioisotope moiety may be detected using PET, thereby allowing the imaging and quantification of integrinexpressing tumor cells as well as the visualization of the distribution, uptake and removal within tumor tissues. The fluorescence imaging agent is used to visualize alphaVbeta3expressing tumor cells and assess the degree of both tumor metastasis and sentinel lymph node (SLN) trafficking. Integrins are transmembrane glycoproteins upregulated on proliferating tumor vessel endothelial cells and various cancer cells; their overexpression has been associated with neovascularization, differentiation, proliferation of tumor cells, metastasis and an overall poor prognosis. 